\title{
Les matériaux multiferroïques
}

\section{I- Le défi du couplage entre magnétisme et ferroélectricité}

Jens Kreise ${ }^{1}$ (Jens.Kreisel@inpg.fr), Wolfgang Kleemann ${ }^{1,2}$, Raphaël Haumont ${ }^{3}$

1 Laboratoire des Matériaux et du Génie Physique (CNRS), INP Grenoble - MINATEC, 38016 Grenoble

2 Angewandte Physik, Universität Duisburg-Essen, 47048 Duisburg, Allemagne

3 Laboratoire de Physico-Chimie de l'État Solide, ICMMO, CNRS, Université Paris XI, 91405 Orsay

L'origine du couplage

de différentes propriétés

physiques au sein

d'un même matériau

est un sujet central

de la physique de la

matière condensée et

interpelle les scientifiques

depuis des siècles.

Nous nous intéressons

ici à une classe de

matériaux qui possèdent

simultanément plusieurs

propriétés dites ferrö̈ques

(ferromagnétisme,

ferroélectricité et/ou

ferroélasticité),

dont le couplage

dépend de la mise en

forme, de la structure

cristallographique

et de l'arrangement

des spins magnétiques,

et constitue un sujet

qui combine un fascinant

défi scientifique avec

un grand potentiel

d'applications.
Un matériau est généralement considéré comme «fonctionnel » s'il possède une propriété physique utilisable dans une application. Actuellement, un grand nombre de recherches se concentrent sur l'étude de matériaux multifonctionnels, dans lesquels plusieurs propriétés peuvent être potentiellement exploitées. Une des questions clés pour le futur développement et la compréhension des matériaux multifonctionnels concerne le couplage mutuel entre les différentes propriétés physiques. Par ailleurs, si un tel couplage existe, il faudra aussi se demander s'il est fort, quels sont les mécanismes mis en jeu, et s'il est exploitable dans des applications.

Afin de répondre à de telles questions, nous nous intéressons particulièrement à une classe de matériaux appelés multiferroiques, qui attirent actuellement un intérêt considérable [1-4]. Les multiferroïques sont des matériaux multifonctionnels par excellence, puisqu'ils possèdent simultanément plusieurs propriétés dites ferröques: ferromagnétisme, ferroélectricité et/ou ferroélasticité. Le préfixe ferro fait historiquement référence au fer, qui montre une aimantation spontanée $M$ qui peut être contrôlée (et notamment être renversée) par un champ magnétique. De la même manière, la polarisation électrique $P$ d'un matériau ferroélectrique est contrôlée par un champ électrique, de même que la déformation ferroélastique $e_{i j}$ l'est par une contrainte mécanique (voir encadré 1, p. 11). La classe des multiferroïques a été élargie aux matériaux présentant des propriétés antiferroïques : antiferromagnétisme, antiferroélectricité. Les matériaux ferroélectriques-ferroélastiques ont été étudiés depuis longtemps et sont à l'origine de nombreuses applications. Un des exemples phares de cette classe est le standard industriel $\mathrm{PbZr}_{1-\mathrm{x}} \mathrm{Ti}_{\mathrm{x}} \mathrm{O}_{3}(\mathrm{PZT})$, dans lequel le couplage entre déformation et polarisation électrique conditionne une forte réponse piézoélectrique qui dépasse largement celle du quartz.

Cependant, les matériaux qui sont à la fois ferroélectriques et ferromagnétiques ont été beaucoup moins étudiés. À ce jour, il n'existe aucune application qui profite d'une telle multifonctionnalité, bien qu'il ait été suggéré, par exemple, que l'aimantation magnétique $M$ et la polarisation électrique $P$ pourraient indépendamment encoder une information dans un seul bit de mémoire. La réelle faisabilité d'une telle mémoire à quatre états (deux magnétiques $M_{\downarrow \uparrow}$ et deux ferroélectriques $\left.P_{\downarrow \uparrow}\right)$ n'a d'ailleurs été démontrée que très récemment [5], et on peut espérer des vitesses de réponse assez grandes pour des applications pratiques. À noter que le fonctionnement d'une telle mémoire ne demande pas de couplage entre ces deux propriétés physiques : un fort couplage serait même désastreux. Au contraire, pour certains matériaux, il faut considérer que l'ordre magnétique et la ferroélectricité puissent être couplés ; la polarisation électrique $P$ serait alors contrôlable par un champ magnétique et l'aimantation $M$ contrôlable par un champ électrique : on parle de couplage magnéto-électrique. Si un tel couplage des deux grandeurs physiques existe au sein d'un matériau à la fois magnétique et ferroélectrique, on peut alors envisager un autre fonctionnement original d'une mémoire : écrire une information électriquement et la lire magnétiquement.

Le peu d'études du passé sur les ferroélectriques-magnétiques s'explique par le fait que ces deux propriétés semblent s'exclure. En effet, la présence d'électrons $d$ des éléments de transition (favorables au magnétisme) défavorise une hybridation avec l'oxygène et donc un déplacement cationique (nécessaire pour la ferroélectricité, voir encadré 1) [6]. De plus, un ferroélectrique doit être isolant pour que les charges mobiles ne détruisent pas la polarisation électrique, alors que la plupart des ferromagnétiques sont des conducteurs. Enfin, si les multiferroïques ferroélectriques-magnétiques se font rares, ceux qui présentent un couplage des deux propriétés - on les appelle multiferroïques magnétoélectriques - le sont encore bien plus. 


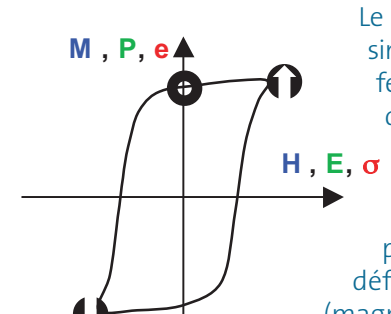

(magnétique, électrique, mécanique). Deux conséquences importantes découlent d'un cycle d'hystérésis : 1) la caractéristique physique reste non nulle - rémanente ou spontanée - en champ nul ( $\mathbf{O}$ ) 2) l'inversion du champ permet de renverser le signe de la caractéristique physique $(\downarrow \uparrow)$.

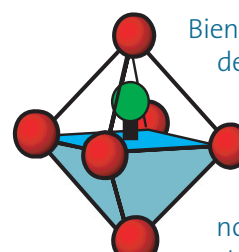

Bien que le phénomène correspondant ait été étudié depuis le $17^{\mathrm{e}}$ siècle, le terme ferroélectricité n'a été introduit qu'en 1912 par Erwin Schrödinger. La ferroélectricité a été longtemps considérée comme un phénomène rare. Mais on connaît aujourd'hui un très grand nombre de matériaux ferroélectriques, notamment sous forme de pérovskites $\mathrm{ABO}_{3}$. Un matériau ferroélectrique est caractérisé par une polarisation électrique spontanée $P$ dont la direction peut être renversée par un champ électrique $E$. Cette polarisation macroscopique est à l'échelle microscopique directement liée à un déplacement des cations chargés positivement (en vert sur la figure) par rapport au barycentre des anions chargés négativement (en rouge)

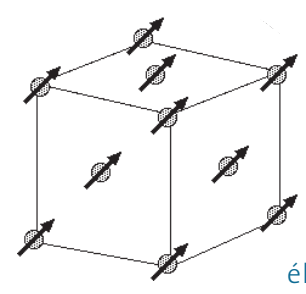

Le ferromagnétisme est la plus ancienne des propriétés ferroïques connues, utilisée par exemple dans la boussole inventée par les Chinois en 100 avant J.C. Un matériau ferromagnétique montre une aimantation $M$ spontanée qui peut être contrôlée par l'application d'un champ magnétique $H$. Le mouvement des électrons dans le nuage électronique est responsable de l'existence d'un magnétisme dit orbital, alors que leur rotation sur eux-mêmes est responsable du magnétisme de spin qui est généralement l'effet prépondérant.

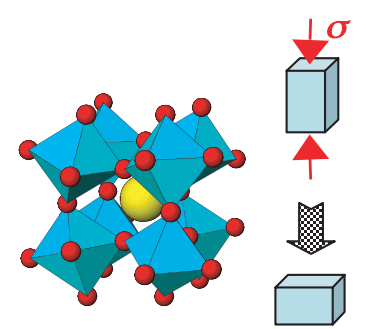

La déformation ferroélastique a été pendant longtemps considérée comme une propriété liée au ferromagnétisme (donnant lieu à la magnétostriction) et à la ferroélectricité (électrostriction). Ce n'est que dans les années 70 que la ferroélasticité a été réellement reconnue comme une propriété indépendante. La figure ci-contre illustre le mécanisme microscopique d'une déformation ferroélastique permanente, même en absence d'une contrainte, à l'exemple de la pérovskite $\mathrm{SrTiO}_{3}$ (en jaune le Sr, en rouge les oxygènes, les Ti étant aux centres des octaèdres). Une contrainte mécanique $\sigma$ peut modifier l'angle et la direction de la rotation des octaèdres (à gauche), et donc l'état de déformation macroscopique e du matériau (à droite). Tout comme pour les autres propriétés ferroïques, un cycle d'hystérésis ferroélastique est ainsi obtenu en appliquant un cycle de contrainte uniaxiale.

\section{Différents matériaux - différents mécanismes}

En conséquence, la recherche de nouveaux systèmes modèles est la condition sine qua non pour mieux comprendre la physique relative au couplage magnétoélectrique et pour envisager d'éventuelles applications. On observe aujourd'hui essentiellement deux démarches pour répondre à cet objectif.

(i) La première démarche, qui connaît actuellement un essor considérable, consiste à construire des multiferrö̈ques artificiels en combinant un matériau ferroélectrique avec un matériau magnétique, sous forme d'une hétérostructure de couches minces ou de diverses formes de composites (multiferroïque extrinsèque). Le lecteur se référera à l'article de Prellier et al. (pp. 14-16) pour plus de détails et les références sur cette démarche.

(ii) La deuxième démarche vise à trouver des nouveaux systèmes multiferrö̈ues intrinsèques, c'est-à-dire des multiferroïques dans lesquels les propriétés ferroélectriques et magnétiques existent au sein du même matériau. Pour répondre à cet objectif, les chercheurs s'intéressent notamment à la synthèse de nouvelles formulations chimiques dans des matériaux sous forme massive ou de couches minces. Le très grand effort de recherche des dernières années a en effet conduit à la découverte de nouveaux matériaux multiferroïques magnéto-électriques. L'étude de ces différents matériaux a notamment permis de mettre en évidence une grande variété de mécanismes de couplage. Discutons deux exemples instructifs pour illustrer comment il est possible d'anticiper qualitativement l'apparition et le type de couplage magnétoélectrique selon le matériau considéré.

Il existe une première famille de matériaux où l'ordre magnétique et l'ordre ferroélectrique sont associés avec deux cations différents. On peut alors anticiper intuitivement que le couplage entre aimantation magnétique et polarisation électrique n'est que faible. Prenons le cas de la pérovskite $\mathrm{BiMnO}_{3}(\mathrm{BMO})$ où le magnétisme est associé aux électrons $3 d$ du $\mathrm{Mn}^{3+}$, alors que la ferroélectricité est conditionnée par le déplacement du cation $\mathrm{Bi}^{3+}$. BMO montre une transition ferroélectrique vers $\mathrm{T}_{F E}=800 \mathrm{~K}$ et une transition ferromagnétique vers $\mathrm{T}_{F M}=110 \mathrm{~K}$, au-dessous de laquelle l'aimantation et la ferroélectricité coexistent. Néanmoins, cette coexistence ne suffit pas à en faire un matériau multiferroïque avec un fort couplage magnéto-électrique : en effet, l'évolution de la constante diélectrique $\varepsilon$ ne montre qu'une très faible anomalie autour de $\mathrm{T}_{F M}$ et elle n'est que très peu dépendante d'un champ magnétique. Aussi, il apparaît vraisemblable qu'un couplage fort nécessite un ingrédient supplémentaire : il faut que le matériau soit également ferroélastique, afin de favoriser un couplage des ordres magnétique et ferroélectrique à travers le sous-système élastique.

Dans un deuxième type de matériaux, la (faible) ferroélectricité n'est pas directement associée à une instabilité structurale, mais est induite indirectement par un ordre de charge et/ou un ordre magnétique particulier, comme illustré sur la figure 1. On considère aujourd'hui que les matériaux avec des dipôles électriques induits par un ordre magnétique présentent potentiellement un couplage magnéto-électrique fort, puisque ces dipôles électriques peuvent être directement ajustés par l'application d'un champ magnétique [7]. En effet, un tel contrôle de propriétés électriques par un champ magnétique a été démontré dans des systèmes à magnétisme frustré, c'est-à-dire des systèmes dans lesquels une compétition des interactions magnétiques empêche la mise en place d'un ordre magnétique simple [7]. Est-ce que l'inverse est vrai ? Malheureusement, les forts effets d'un champ magnétique sur la polarisation électrique et sur la constante diélectrique ne conditionnent pas forcément 

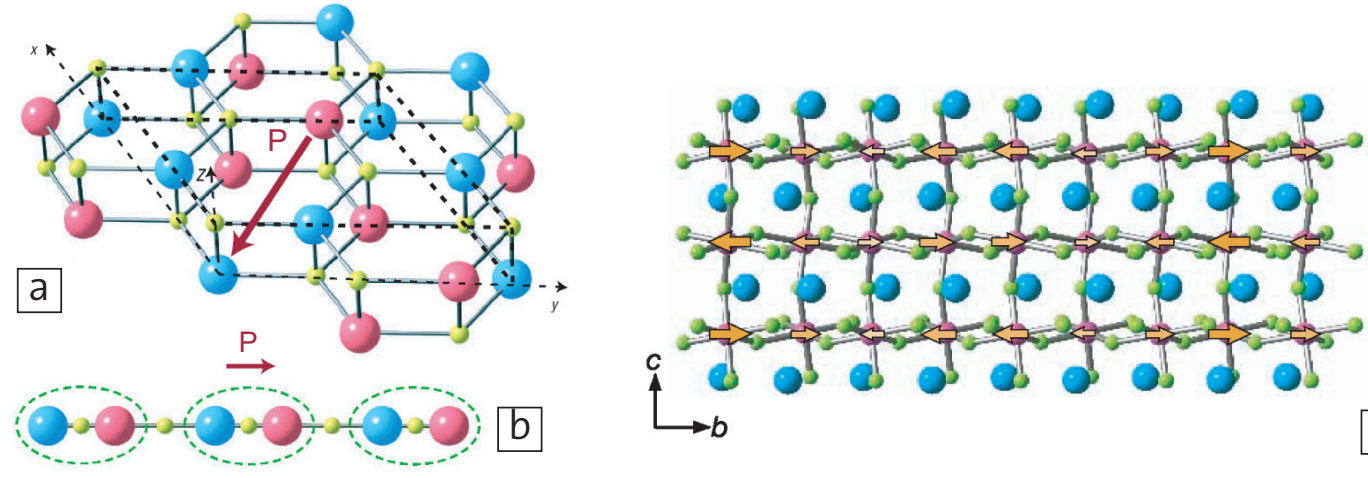

Moment magnétique du Mn

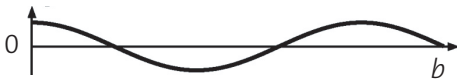

Déplacement atomique du Mn $\Delta z$ C

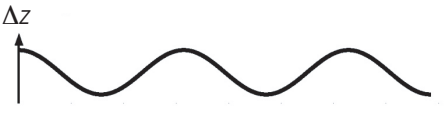

Figure 1: Ordre de charge et structure magnétique comme origine de ferroélectricité.

a,b) Illustration schématique d'une ferroélectricité induite par deux types d'ordre de charge (les cations rouges et bleus portent une charge positive plus ou moins importante). La flèche rouge représente le vecteur de polarisation électrique $P$ résultant du déplacement atomique induit par l'ordre de charge. [Figure d'après S-W. Cheong et M. Mostovoy, Nature Materials 6 (2007) 13.]

c) Illustration d'une modulation de la ferroélectricité par une structure magnétique. La figure de gauche représente schématiquement la structure cristalline de $\mathrm{TbMnO}_{3}$ (en bleu : $\mathrm{Tb}$, en rouge: $M n$, en vert : O) et la modulation de la structure magnétique (direction et taille des flèches oranges). Les deux figures de droite illustrent la corrélation entre la variation du moment magnétique du cation Mn et son déplacement ferroélectrique selon l'axe $b$ du composé. [Figure d'après T. Kimura et al., Nature 426 (2003) 55]

\section{Références}

\section{[1] M. Fiebig, J. Phys. D:Appl.}

Phys. 38 (2005) R1-30.

[2] W. Eerenstein et al., Nature 442 (2006) 759

[3] W. Kleemann \& J. Kreisel (eds), "Current Progress in Multiferroics and Magnetoelectrics", Phase

Transitions 79 (12) (2006)

(special issue).

[4] N. Mathur \& M. Bibes (eds), "Multiferroic and magnetoelectric epitaxial thin films and devices", Philosophical Magazine 87 (3-4) (2007) (special issue).

[5] M. Gajek et al., Nature materials 6 (2007) 296-302.

[6] N.A. Hill, J. Phys. Chem. B 104 (2000) 317-338.

[7] S.W. Cheong \& M. Mostovoy, Nature materials 6 (2007) 13-20. [8] A.B. Sushkov et al., Phys. Rev. Lett. 98 (2007) 027202.

[9] P. Borisov et al., Phys. Rev. Lett. 94 (2005) 117203.

[10] X. Chen et al., Appl. Phys. Lett. 89 (2006) 202508. une forte dépendance des propriétés magnétiques envers un champ électrique. En effet, des exemples pour lesquels une structure magnétique complexe peut être contrôlée par un champ électrique restent très rares. Néanmoins, de très récents résultats montrent qu'un champ électrique peut, dans certains cas, contrôler le sens - horaire ou anti-horaire d'une hélice magnétique, suggérant que l'on peut encore s'attendre à des effets tout à fait surprenants.

Les deux exemples ci-dessus illustrent comment il est possible, qualitativement, d'anticiper un couplage magnéto-électrique dans un matériau. Néanmoins, il faut souligner que l'existence ou non d'un tel couplage ne peut formellement être comprise et prévue que par des considérations de symétrie (structurale et magnétique). Une telle analyse permet de prévoir si le couplage est direct (linéaire ou non linéaire) ou indirect par le sous-système élastique (électrostriction/piézoélectricité ou magnétostriction/piézomagnétisme) - voir l'encadré 2 pour plus de détails.

Un des défis actuels des multiferrö̈ques magnéto-électriques est l'observation expérimentale du couplage. Parmi toutes les approches explorées, la recherche d'une excitation fondamentale, dite électromagnon, qui résulterait directement du couplage magnéto-électrique, parait particulièrement intéressante. L'existence d'un électromagnon permettrait notamment de mieux estimer les constantes du couplage magnéto-électrique, similairement aux magnons pour le magnétisme ou encore aux vibrations du réseau (phonons) pour les distorsions ferroélectriques ou ferroélastiques. Bien que déjà prédits théoriquement dans les années 80 , ce n'est que récemment [8] que de tels électromagnons ont été observés.

\section{Applications multiferroïques ou magnéto-électriques - Réalité et défis}

La rareté des matériaux multiferroïques magnéto-électriques n'a pas découragé les chercheurs à réfléchir sur leurs applications potentielles en "spintronique " (technologie utilisant les spins et leurs moments magnétiques plutôt que les charges des électrons).

D'un côté, les travaux récents [5] sur le multiferroïque $\mathrm{Bi}_{0,9} \mathrm{La}_{0,1} \mathrm{MnO}_{3}$ (ferromagnétique-ferroélectrique, mais sans couplage magnéto-électrique) ont ouvert le chemin pour l'introduction d'une logique quaternaire, qui a le potentiel de révolutionner la technologie des ordinateurs. Néanmoins, un sévère désavantage de ce système reste sa température de Curie ferromagnétique trop basse, $\mathrm{T}_{\mathrm{c}} \approx 100 \mathrm{~K}$.

Par ailleurs, certains matériaux magnétoélectriques (mais pas multiferroïques) offrent un

\section{"Coupler ou ne pas coupler, telle est la question...."}

\section{ou « Le rôle éminent de la symétrie "}

La multifonctionnalité des matériaux magnéto-électriques (ME) est basée sur la relation entre les champs électrique $\boldsymbol{E}$ et magnétique $\boldsymbol{M}$ et les propriétés physiques des matériaux, respectivement la polarisation $P$ et l'aimantation $\boldsymbol{M}$

$$
P_{i}^{M E}=\chi_{i j}^{E M} H_{j} \quad \text { et } \quad M_{i}^{M E}=\chi_{i j}^{M E} E_{j} \quad \text { où } \quad \chi_{i j}^{E M}=\chi_{j i}^{M E} \quad \text { sont les tenseurs des susceptibilités. }
$$

Il a été montré qu'une susceptibilité ME non nulle n'est autorisée que dans peu de cristaux magnétiques, possédant un groupe ponctuel particulier. De façon générale, ni une symétrie d'inversion ni une symétrie d'espace-temps ne sont autorisées pour un matériau magnéto-électrique. En conséquence, un système magnéto-électrique est souvent polairement et magnétiquement ordonné, comme par exemple l'antiferromagnétique trigonal $\mathrm{Cr}_{2} \mathrm{O}_{3}$. Dans les cas où la symétrie n'autorise pas l'existence d'un couplage direct, les termes de couplage d'ordres supérieurs interviennent. De façon générale, ces termes sont souvent inférieurs aux termes linéaires ; aussi, le couplage s'effectue plutôt par le biais d'un couplage indirect contrainte-tension, c'est-à-dire que les ordres ferroélectrique et ferromagnétique se produisent par et s'accompagnent de contraintes électrostrictives et magnétostrictives. Ce mécanisme est tout aussi valable pour les multiferrö̈ques intrinsèques que pour les multiferroïques composites - lesquels sont constitués d'une phase ferroélectrique (électrostrictive) mêlée intimement à une phase ferromagnétique (magnétoélastique). 


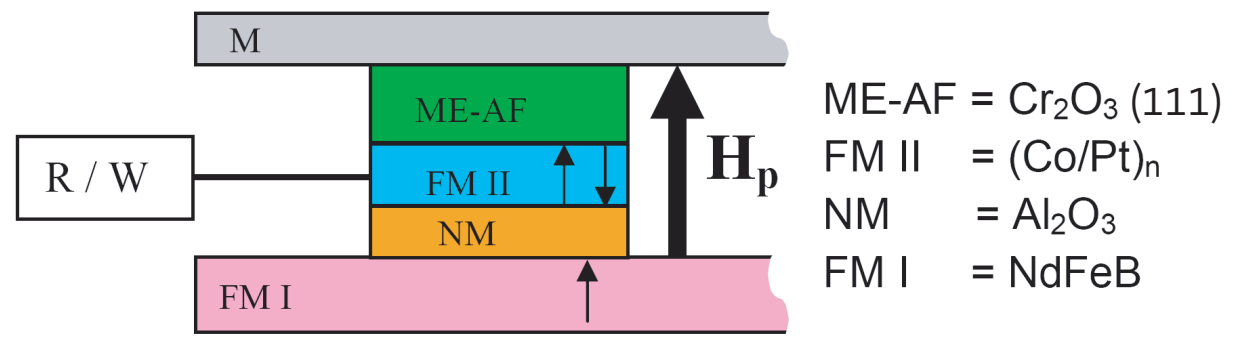

chemin alternatif. Un des matériaux prometteurs est l'oxyde $\mathrm{Cr}_{2} \mathrm{O}_{3}$, qui n'est ni ferromagnétique ni ferroélectrique, mais magnéto-électrique et antiferromagnétique en dessous de sa température de Néel, $T_{\mathrm{N}}=307 \mathrm{~K}$. De façon surprenante, cette combinaison de propriétés est avantageuse : un refroidissement magnéto-électrique (application simultanée d'un champ électrique et d'un champ magnétique à une température inférieure à $T_{\mathrm{N}}$ ) conduit à un domaine antiferromagnétique qui peut tourner l'aimantation d'un dépôt ferromagnétique "up" et "down”, selon l'arrangement de ses spins par des interactions d'interfaces ("exchange bias") [3, 9]. La figure 2 illustre l'architecture d'une mémoire RAM magnétoélectrique (MERAM) basée sur ce principe, et qui permet de répondre à la demande d'une dissipation minimale de chaleur des futures mémoires RAM à haute densité et à trois dimensions.

\section{Défis futurs}

La vraie solution d'un composant multiferroïque à fort couplage magnéto-électrique n’a pas encore été trouvée et ce défi va continuer à motiver des chercheurs du monde entier, toujours à la recherche du "Graal ". Certains chercheurs voient le multiferroïque historique $\mathrm{BiFeO}_{3}$ [3] comme le "Graal magnétoélectrique ", mais ce matériau n'est qu'antiferromagnétique. Cependant, sa remarquable polarisation ferroélectrique fait de $\mathrm{BiFeO}_{3}$ un matériau phare de la dernière génération des mémoires ferroélectriques (Ferroelectric RAM FER AM). Concernant son potentiel magnétique, $\mathrm{BiFeO}_{3}$ sera probablement dépassé par des matériaux dérivés d'un composé chimiquement similaire : $\mathrm{BiMnO}_{3}$.

La demande d'une symétrie suffisamment basse pour permettre la magnéto-électricité implique une symétrie cristalline et une structure de spin particulières. En conséquence, il ne serait pas surprenant de découvrir des matériaux désordonnés qui répondraient aux espoirs de trouver un couplage magnéto-électrique à température ambiante, et pourquoi pas même à une température bien supérieure. Un autre espoir réside sur les avancées faites dans le domaine des multiferroïques artificiels, comme l'expose W. Prellier dans l'article suivant.
Fig.2 : Architecture d'une mémoire RAM magnéto-électrique (MERAM). Le champ magnétique $H_{p}$ et l'aimantation de la couche ferromagnétique épaisse (FM I) restent "up" en permanence. Pour écrire, une tension électrique positive ou négative est appliquée entre la ligne lire/ écrire $(R / W)$ et l'électrode métallique $M$. L'information (" $O$ " ou " 1 ") est stockée dans la structure de domaine antiferromagnétique de la couche ME-AF de $\mathrm{Cr}_{2} \mathrm{O}_{3}$, qui joue le rôle de mémoire, et qui contrôle l'aimantation de la multicouche ferromagnétique FM II, parallèle ou antiparallèle à celle de FM I. Les données sont lues, tout comme dans une RAM magnétique, en mesurant la magnétorésistance "géante " de valeur basse ("O") ou élevée ("1"), respectivement, entre la ligne $R / W$, le long de l'électrode $F M I I$, une couche non magnétique isolante (NM) et l'électrode FM I. On peut s'attendre à un temps de réponse en dessous de 100 ns, en utilisant des cellules MERAM de taille $130 \mathrm{~nm}$. Pour plus de détails, voir Chen et al. [10].

Remerciements

Nos recherches sur les matériaux multiferroïques sont effectuées dans le cadre de différents réseaux et actions.

JK remercie le réseau européen d'excellence FAME (www.famenoe.org/), le STREP européen $\mathrm{MaCoMuFi}$ (www.macomufi.eu/) et l'ANR pour le soutien financier. WK remercie le STREP européen Multiceral (http://multiceral.web.ua.pt/).

Ce manuscrit a été préparé pendant le séjour sabbatique de WK en tant que professeur invité à l'INP Grenoble. 